\title{
Polariton lasing by exciton-electron scattering in semiconductor microcavities
}

\author{
G. Malpuech, ${ }^{1,3}$ A. Kavokin, ${ }^{2}$ A. Di Carlo, ${ }^{1}$ and J. J. Baumberg ${ }^{3}$ \\ ${ }^{1}$ INFM-Department of Electrical Engineering, University of Rome "Tor Vergata", Via di Tor Vergata 110-I-00133 Roma, Italy \\ ${ }^{2}$ LASMEA, CNRS-Universite Clermont-II "Blaise Pascal” 24 Avenue des Landais, 63177 Aubiere Cedex, France \\ ${ }^{3}$ Department of Physics \& Astronomy, University of Southampton, SO17 1BJ Southampton, United Kingdom
}

(Received 20 December 2001; published 1 April 2002)

\begin{abstract}
The relaxation bottleneck present in the dispersion relation of exciton polaritons in semiconductor microcavities has prevented the realization of low threshold lasing based on exciton-polariton condensation. Here we show theoretically that the introduction of a cold electron gas into such structures induces efficient electronpolariton scattering. This process allows the condensation of the polaritons accumulated at the bottleneck to the final emitting state with a transition time of a few picoseconds, opening the way to a new generation of low-threshold light-emitting devices.
\end{abstract}

DOI: 10.1103/PhysRevB.65.153310

PACS number(s): 78.47.+p, 42.50. $-\mathrm{p}, 42.65 .-\mathrm{k}, 71.36 .+\mathrm{c}$

The observation of the strong coupling of light with excitons in semiconductor microcavities ${ }^{1}$ has generated much speculation regarding the possibility for low-threshold optical devices. ${ }^{2,3}$ Realization of such devices based on the Bosonic character of the optical eigenmodes (exciton polaritons ${ }^{4}$ ) of these structures would be a revolutionary step in semiconductor optics. However, Bose condensation of exciton polaritons has not yet been observed. One of the main obstacles is set by a bottleneck in the polariton relaxation rate. ${ }^{5-7}$ As a result, the emission of the microcavity under nonresonant excitation remains weak and nondirectional.

Here we propose an original relaxation mechanism based on the scattering of polaritons with free electrons. This allows polaritons to relax efficiently from the bottleneck region to the lowest energy state once free electrons are introduced into the active region either via doping or by photoexcitation. This opens the way to realization of lowthreshold laserlike devices based on cavity polaritons.

The basic principle of a polariton laser is illustrated in Fig. 1(a) using the dispersion curve of the lower branch exciton polaritons in a typical microcavity. The strong-coupling regime creates a trap containing a small number of polariton states at energies below all other states in the semiconductor. This polariton trap is sharp with a depth equal to nearly half the splitting $\Omega$ between the two polariton modes. Polaritons in the trap are half photon and half exciton, and have properties suitable for the Bose condensation of exciton polaritons once sufficiently populated. Recombination from this state in the Bose condensation regime is coherent, monochromatic, and sharply directed, characteristic of laser emission. The relaxation of polaritons into the $k=0$ state is found to be stimulated if the population of the final state is larger than $1 .{ }^{8}$

The amplification process proposed here is physically very different from the classical lasing process. ${ }^{9}$ In particular, the threshold to lasing which in conventional lasers is conditional on the inversion of population is only dependent on the lifetime of the ground state in the polariton laser. As soon as relaxation to the ground state of the trap becomes faster than the radiative recombination from this state, optical amplification is achieved. Note that the absorption and re-absorption of light are intrinsically taken into account within the polariton picture (yielding the renormalized eigenmode energies) while in a conventional laser the absorption channel must be balanced by stimulated emission that requires the inversion of population. Strictly speaking, in a polariton laser, inversion of population is not required. Hence the threshold pump powers and energy consumption of polariton lasers are expected to be extremely low, even compared to VCSEL's. ${ }^{10}$

The major problem to be resolved on the way towards realization of polariton lasers comes from a relaxation bottleneck $^{5,6}$ in the dispersion relation. For small in-plane wave vectors acoustic phonons cannot assist in the polariton relaxation and polaritons accumulate in a broad metastable region which is scarcely coupled to light external to the device. Nevertheless, in recent years new experimental results have appeared giving hope for realization of polariton lasers. Under nonresonant excitation, strong superlinear photoluminescence of the lower polariton branch has been seen both in II-VI (Ref. 11) and III-V (Ref. 12) heterostructures. Under resonant excitation, the high efficiency of pair scattering processes between polaritons at the so-called magic angle ${ }^{13}$ has been shown under pulsed excitation, ${ }^{8,14,15} \mathrm{cw}$ excitation, ${ }^{16,17}$ and theoretically. ${ }^{18}$ These results clearly demonstrate that
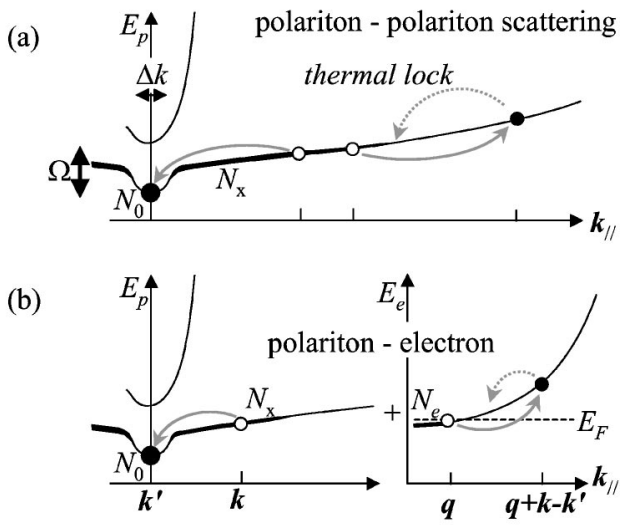

FIG. 1. (a) Dispersion relations of upper and lower polaritons, showing the dominant pair scattering of polaritons feeding energy into the trap at $k=0$. (b) Similar polariton-electron scattering process. The excited carriers relax quickly preventing re-ionization. 
cavity polaritons behave as bosons and that they can condense in the trap if a resonant scattering process is available. However, polariton-polariton scattering is only efficient for resonant optical pumping of the cavity.

In this paper a different scattering mechanism is proposed based on the interactions of polaritons and free electrons. ${ }^{19}$ We show that if a concentration of free electrons is introduced into the structure, polaritons can be efficiently scattered from the bottleneck region to the ground state [Fig. 1(b)]. Through this process, a polariton passes its excess energy and wave vector to a free electron, as described by

$$
\begin{aligned}
& e^{-}(\mathbf{q})+P(\mathbf{k}) \rightarrow e^{-}\left(\mathbf{q}+\mathbf{k}-\mathbf{k}^{\prime}\right)+P\left(\mathbf{k}^{\prime}\right), \\
& E\left(\mathbf{k}^{\prime}\right)+\frac{\hbar^{2}\left(\mathbf{q}+\mathbf{k}-\mathbf{k}^{\prime}\right)^{2}}{2 m_{e}}-\frac{\hbar^{2} \mathbf{q}^{2}}{2 m_{e}}=E(\mathbf{k}),
\end{aligned}
$$

where $e^{-}$denotes an electron, $E$ is the polariton energy, and $m_{e}$ is the electron effective mass. In a typical experimental configuration, the net heating of the electron gas is minimal because the hot electrons are rapidly cooled by the reservoir of cold electrons present in the QW. The polaritons cannot be re-ionized because the hot electrons relax so rapidly, thus providing a "thermal lock" for the trapped polaritons. This thermal lock is a key difference with population dynamics in a normal laser, and a characteristic of two-particle stimulated transitions. While the exciton polaritons are excited within an area of only $10^{-8} \mathrm{~cm}^{2}$, the cold electron gas can dissipate heat by lateral thermal diffusion over the entire sample. The coexistence of excitons and free electrons in a quantum well is possible at intermediate free charge densities (see, e.g., Ref. 20). An additional effect that can modify the cavity dispersion in this regime is the appearance of a trion state coupled to the cavity modes. ${ }^{22}$ In this case the stimulated scattering of polaritons can be accompanied by dissociation of trions while the effect remains qualitatively similar. Further we shall neglect the trions for simplicity.

The advantage of this scattering mechanism with respect to previously discussed ones is that (i) the matrix element of electron-exciton scattering is quite large, ${ }^{21,22}$ (ii) the electron has a much lighter mass than an exciton, thus the energy relaxation of a polariton from the bottleneck region to the ground state requires fewer scattering events than for polariton-polariton or polariton-phonon scattering. These advantages are found to be strong enough to restore fast polariton relaxation and allow the polaritons to condense into their traped state.

We simulate the relaxation of cavity polaritons excited nonresonantly in a continuous regime. A Boltzmann equation is solved numerically in two-dimensional reciprocal space:

$$
\begin{aligned}
\frac{d n_{k}}{d t}= & P_{k}-\Gamma_{k} n_{k}-n_{k} \sum_{k^{\prime}} W_{k \rightarrow k^{\prime}}\left(n_{k^{\prime}}+1\right) \\
& +\left(n_{k}+1\right) \sum_{\mathbf{k}^{\prime}} W_{\mathbf{k}^{\prime} \rightarrow \mathbf{k}^{\prime}} n_{k^{\prime}},
\end{aligned}
$$

where $n_{k}$ is the polariton distribution function in the state having a wave vector $\mathbf{k}, P_{k}$ is the generation term describing optical pumping. $\Gamma_{k}$ is the recombination rate composed of both radiative and nonradiative components. $W_{k \rightarrow k^{\prime}}$ is the total scattering rate between the states $\mathbf{k}$ and $\mathbf{k}^{\prime}$. This scattering rate is comprised of three terms in our model, namely: polariton-acoustic phonon scattering (rate $W_{a}$ ), polaritonpolariton scattering (rate $W_{x}$ ), and polariton-electron scattering $\left(\right.$ rate $W_{e}$ ). We assume perfect cylindrical symmetry for the polariton distribution function, implying that the population of a state $\mathbf{k}$ is only a function of the absolute value of the wave vector, relevant to the case of nonresonant pumping. Only the intrabranch scattering within the lower polariton branch is considered. We calculate the radiative recombination rate, polariton-acoustic phonon, and the polaritonpolariton scattering rates as in Ref. 5 . The cavity photon lifetime $\tau_{c}$ is taken to be $8 \mathrm{ps}$, whereas the radiative lifetime of the QW excitons is $20 \mathrm{ps}$. The polariton nonradiative lifetime is chosen constant over the whole reciprocal space (1 ns). The polariton-electron scattering rate is calculated using the Fermi golden rule as

$$
\begin{aligned}
W_{\mathbf{k} \rightarrow \mathbf{k}^{\prime}}^{e}= & \frac{2 \pi}{\hbar} \sum_{\mathbf{q}} M^{2} N_{\mathbf{q}}\left(1-N_{\mathbf{q}^{+\mathbf{k}^{\prime}}-\mathbf{k}}\right) \\
& \times \delta\left(E\left(\mathbf{k}^{\prime}\right)-E(\mathbf{k})-\frac{\hbar^{2}}{2 m_{e}}\left(\mathbf{q}^{2}-\left|\mathbf{q}+\mathbf{k}-\mathbf{k}^{\prime}\right|^{2}\right)\right),
\end{aligned}
$$

where $q$ is the wave vector of an electron required for the resonant scattering, and $N_{\mathbf{q}}$ is the Fermi-Dirac electron distribution function. Initially, an electron concentration $n_{e}$ $=10^{10} \mathrm{~cm}^{-2}$ and a lattice temperature of $10 \mathrm{~K}$ are assumed. $M$ is the matrix element of interaction between an electron and a polariton. $M=M_{d i r} \pm M_{e x c}$, corresponding to the triplet configuration $(+$, parallel electron spins), and the singlet configuration (-, antiparallel electron spins). Further, we shall only include the stronger electron-polariton scattering of the triplet configuration. In the Born approximation,

$$
\begin{gathered}
M_{d i r}=\frac{2 \pi e^{2}}{A \varepsilon} \frac{x_{k} x_{k^{\prime}}}{\left|\mathbf{k}-\mathbf{k}^{\prime}\right|}\left(\left[1+\zeta_{h}^{2}\right]^{-3 / 2}-\left[1+\zeta_{e}^{2}\right]^{-3 / 2}\right), \\
M_{e x}=\frac{8 \pi e^{2}}{A \varepsilon} \frac{x_{k} x_{k^{\prime}}\left(\left[1+\zeta_{c}^{2}\right]^{-3 / 2}-\left[1+4 \zeta_{h}^{2}\right]^{-3 / 2}\right)}{\left[a^{-2}+\left|\mathbf{q}-\beta_{e} \mathbf{k}^{\prime}\right|^{2}\right]^{1 / 2}},
\end{gathered}
$$

$\varepsilon$ is the dielectric susceptibility of the QW, $x_{k}$ is the exciton fraction of the polariton state having a wave vector $\mathbf{k}, \zeta_{e, h}$ $=\frac{1}{2} \beta_{e, h}\left|\mathbf{k}-\mathbf{k}^{\prime}\right| a, \zeta_{c}=\left(\beta_{e} \mathbf{k}+\left|\mathbf{k}-\mathbf{k}^{\prime}\right|-\mathbf{q}\right) a, \beta_{e, h}=m_{e, h} /\left(m_{e}\right.$ $+m_{h}$ ) where $m_{e}$ and $m_{h}$ are electron and hole effective masses, respectively, $a$ is the in-plane Bohr radius of the QW exciton, and $A$ is a normalization area.

Figure 2 shows the equilibrium distribution function under continuous pumping for a GaAs-based cavity having a normal-mode splitting of $5 \mathrm{meV}$ containing a single QW, and for zero detuning of cavity and exciton modes. Here we use throughout these parameters of the microcavity sample of Ref. 15. For all the curves the pump power absorbed by the single $\mathrm{QW}$ is set to be $4.2 \mathrm{~W} / \mathrm{cm}^{2}$ between $k=3$ $\times 10^{6} \mathrm{~cm}^{-1}$ and $k=5 \times 10^{6} \mathrm{~cm}^{-1}$ roughly equivalent to an excess of energy of $20 \mathrm{meV}$. Note that the results we present 


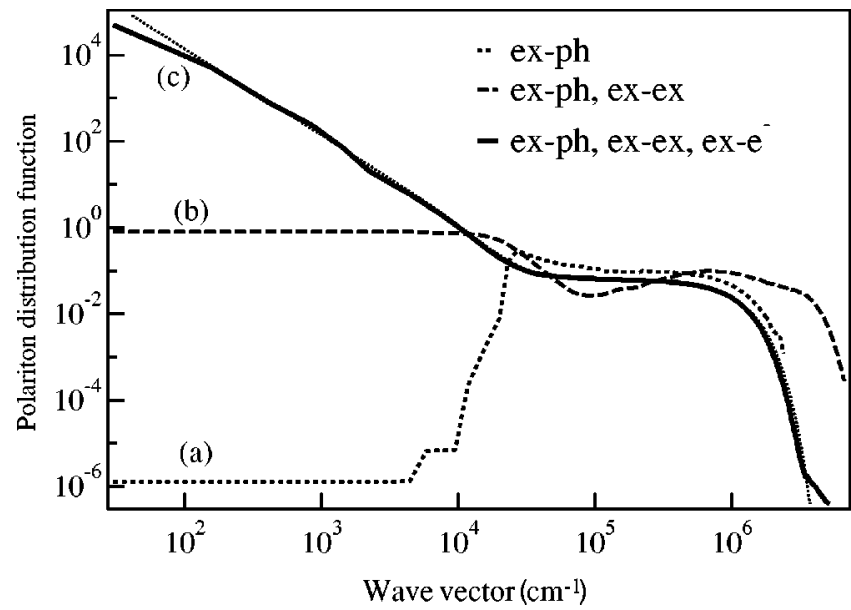

FIG. 2. Distribution function of polaritons at $10 \mathrm{~K}$ when nonresonantly pumped with a power of $4.2 \mathrm{~W} / \mathrm{cm}^{2}$. Results are shown for (a) polariton-acoustic phonon scattering (dotted), (b) as (a) plus polariton-polariton scattering (dashed), and (c) as (b) plus polaritonelectron scattering (solid). The thin dotted line shows the equilibrium Bose distribution function with zero chemical potential.

are not strongly sensitive to the pumping conditions, which allows for both optical or electrical pumping mechanisms. Taking into account only the acoustic-phonon scattering [curve (a) in Fig. 2], a thermalized distribution function is seen only beyond $k=2 \times 10^{4} \mathrm{~cm}^{-1}$ (the bottleneck region) where the polaritons accumulate. Equilibrium is reached after $10 \mathrm{~ns}$ leaving a polariton density of $2.5 \times 10^{10} \mathrm{~cm}^{-2}$. Including both polariton-polariton and polariton-acoustic phonon scattering processes [curve (b) in Fig. 2] shows partial relaxation of the bottleneck and a flat polariton distribution. However, the equilibrium polariton density in the cavity remains the same, close to the saturation density for excitons $\left(\sim 5 \times 10^{10} \mathrm{~cm}^{-2}\right)$. The distribution function near the polariton ground state approaches 1 . This result is in excellent agreement with experimental results obtained up to now ${ }^{7,23}$ which shows that the threshold amplification for the distribution function at the trap state is reached when the strongcoupling regime is likely to be destroyed. To get an increase in the population of the lowest $k$ state one has to increase the population of a large number of states requiring a large density of excitons. This is due to the flat shape of the distribution function which comes from the nature of the polaritonpolariton scattering process-each scattering event increases the population of the high- $k$ states. The relaxation of the polaritons from these states is then assisted only by phonons and is slow. The radiative efficiency, which we estimate as the ratio of photons leaving the cavity within a cone of less than $1^{\circ}$ to the pump power, is thus only $1.7 \%$.

When all three scattering processes listed above are taken into account [curve (c) in Fig. 2], a huge occupation number of the lowest energy state of more than $10^{4}$ is achieved. This system thus acts as a polariton laser in that the scattering of polaritons injected at high $k$ by optical or electrical pumping is stimulated by the population of the low- $k$ states. In this situation the light power emitted in a cone of $1^{\circ}$ is $3.3 \mathrm{~W} / \mathrm{cm}^{2}$ and the efficiency of the energy transfer from
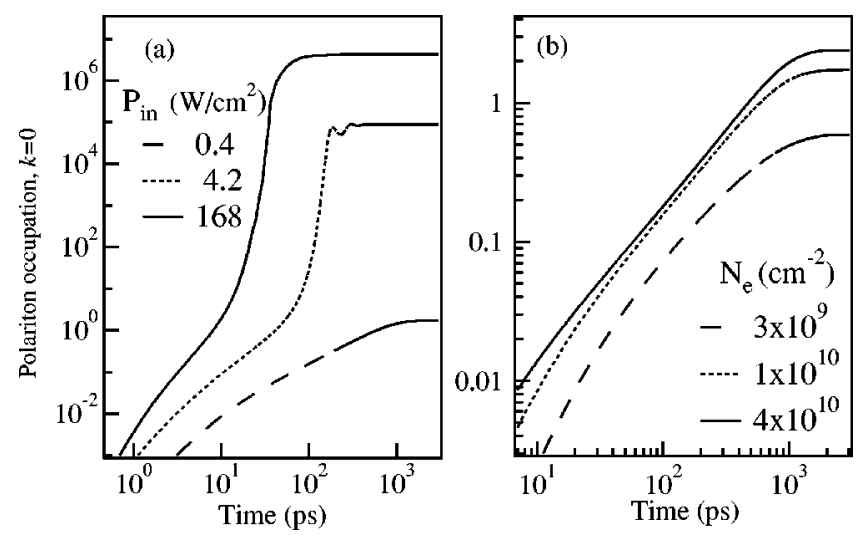

FIG. 3. (a) Polariton occupation of the $k=0$ state vs time for excitation powers of $0.42 \mathrm{~W} / \mathrm{cm}^{2}$ (dashed), $4.2 \mathrm{~W} / \mathrm{cm}^{2}$ (dotted), $168 \mathrm{~W} / \mathrm{cm}^{2}$ (solid). The pump is turned on at $t=0$. The corresponding total polariton equilibrium densities are $7.0 \times 10^{8}, 1.3$ $\times 10^{9}$, and $1.3 \times 10^{10} \mathrm{~cm}^{-2}$. (b) As (a) for a pump power of $0.42 \mathrm{~W} / \mathrm{cm}^{2}$ and electron doping of $2.5 \times 10^{9} \mathrm{~cm}^{-2}$ (dashed), $10^{10} \mathrm{~cm}^{-2}$ (dotted), $4 \times 10^{10} \mathrm{~cm}^{-2}$ (solid).

pump to emitted light is about $80 \%$. The light emitted by the cavity is much more directional and comes from a smaller number of states that in case (b). The equilibrium polariton density in the cavity is now $1.25 \times 10^{9} \mathrm{~cm}^{-2}$, i.e., 20 times lower than in cases (a) and (b). Pump powers at least 40 times stronger can be used before the strong- to weakcoupling threshold is exceeded. The thin dotted line in Fig. 2 shows the equilibrium polariton density from a Bose distribution function plotted for zero chemical potential. The good agreement with curve (c) clearly demonstrates that a thermodynamic equilibrium is achieved for this value of the chemical potential, that is a signature of Bose condensation of polaritons.

This is confirmed by tracking the temporal evolution of the distribution function at the ground state for different excitation powers [Fig. 3(a)]. For the strongest pumping, it is strongly enhanced at very short times as soon as it exceeds 1 , demonstrating the Bose amplification of the final-state population. The dashed curve corresponds to the threshold condition for Bose amplification, producing an equilibrium population of the $k=0$ state slightly exceeding 1 , but without significant amplification occurring.

Figure 3(b) shows the temporal evolution of the population of the ground state for different doping. The equilibrium density is strongly enhanced and exceeds the lasing threshold for electron densities above $10^{10} \mathrm{~cm}^{-2}$. Figure 4 shows the radiative efficiency of the device as a function of input power with (a) and without (b) polariton-electron scattering. In the first case, the emission rises quadratically up to the threshold, while in the second case it is much larger and linearly increases. The dotted line on the curve (b) marks the excitation conditions for which the strong-coupling regime collapses because of the bleaching of the excitons. For the doped microcavity, an excitation area of diameter $100 \mu \mathrm{m}$ corresponds to an extremely low threshold power of $3 \mu \mathrm{W}$. However, high excitation powers can also be used, while conserving the strong-coupling regime.

Finally, we address the effect of heating of the electron 


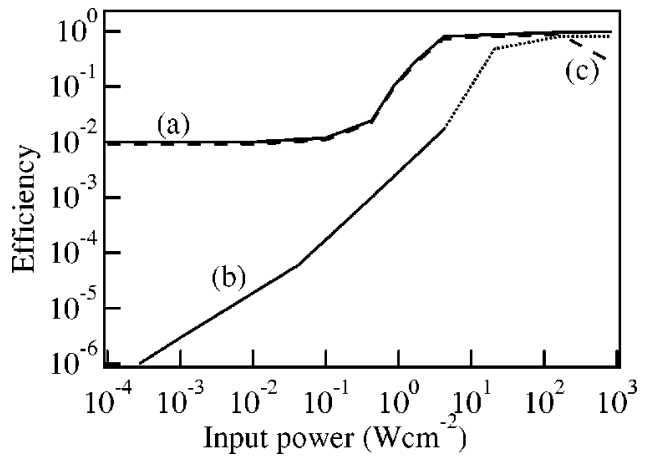

FIG. 4. Radiative efficiency vs power absorbed in the microcavity at $10 \mathrm{~K}$, for (a) a doped cavity, $n_{e}=10^{10} \mathrm{~cm}^{-2}$, (b) an undoped cavity, and (c) as (a) but including the effects of electron-gas heating. The dotted part of curve (b) corresponds to a calculated exciton density $>5 \times 10^{10} \mathrm{~cm}^{-2}$.

gas due to the electron-exciton scattering on the characteristics of the polariton laser. The dashed line in Fig. 4 shows the radiative efficiency of the polariton laser calculated taking into account the dynamics of free electrons. The calculation simultaneously solves the Boltzmann equation for the exciton polaritons [Eq. (3)] together with the Boltzmann equation for the electron gas taking into account the electron-electron, electron-polariton, and electron-acoustic phonon scattering processes. One can see that the result is identical to the curve obtained for the equilibrium Fermi distribution of electrons for pump intensities below $100 \mathrm{~W} / \mathrm{cm}^{2}$. This is because of efficient electron-electron scattering (rate $\sim 1.5 \mathrm{ps}^{-1}$ in our case) that leads to rapid relaxation of the electron gas to its thermal equilibrium. At pump intensities of $1000 \mathrm{~W} / \mathrm{cm}^{2}$ the temperature of the electron gas is $22 \mathrm{~K}$, approaching the critical temperature for Bose condensation of polaritons in our system $(25 \mathrm{~K})$. At higher pumping intensities, the electron-polariton interaction starts to play an important role, and the radiative efficiency of the polariton laser decreases due to the ionization of the polaritons from their ground state by hot electrons.

In conclusion, we have shown theoretically that exciton polaritons continuously injected into a doped microcavity reach their thermodynamic equilibrium and Bose condense thanks to the presence of a two-dimensional electron gas in a microcavity. Lasing in the strong-coupling regime of these semiconductor microcavities is simulated and the threshold found is dramatically lower than in typical VCSEL's. The proposed effect opens a way towards realization of a new generation of low-threshold light-emitting devices based on the Bose condensation of microcavity polaritons. To make such polariton devices operate at room temperature, the use of large band-gap semiconductors $(\mathrm{GaN}, \mathrm{ZnO})^{24}$ or of organic materials seems to be the most promising way forward.

This work has been supported by the European Commission in the framework of the contract CLERMONT HPRNCT-1999-00132, HEFCE JR98SOBA, FMRX-CT970134, and EPSRC GR/N18598. One of us (A.D.C.) acknowledges the support of ONR.
${ }^{1}$ C. Weisbuch et al., Phys. Rev. Lett. 69, 3314 (1992).

${ }^{2}$ A. Imamoglu et al., Phys. Rev. A 53, 4250 (1996).

${ }^{3}$ J.J. Baumberg et al., Phys. Rev. B 62, R16 247 (2000).

${ }^{4}$ Exciton polaritons are the eigenmodes of propagation of the light in semiconductors in the vicinity of exciton resonances. For a review, see, e.g., L.C. Andreani, in Confined Electrons and Photons, edited by E. Burstein and C. Weisbuch (Plenum Press, New York, 1995), p. 57.

${ }^{5}$ F. Tassone et al., Phys. Rev. B 56, 7554 (1997); 59, 10830 (1999).

${ }^{6}$ A.I. Tartakovskii et al., Phys. Rev. B 62, R2283 (2000).

${ }^{7}$ P. Sellenart et al., Phys. Rev. B 62, R16 263 (2000).

${ }^{8}$ P. Savvidis et al., Phys. Rev. Lett. 84, 1547 (2000).

${ }^{9}$ T.H. Maiman, Nature (London) 187, 493 (1960); A.L. Schawlow et al., Phys. Rev. 112, 1940 (1958).

${ }^{10}$ T.E. Sole, Vertical Surface Emitting Lasers (Wiley, New York, 1995).

${ }^{11}$ Le Si Dang et al., Phys. Rev. Lett. 81, 3920 (1998).

${ }^{12}$ P. Senellart et al., Phys. Rev. Lett. 82, 1233 (1999).
${ }^{13}$ The magic angle corresponds to a particular point of the polariton dispersion relation for which the pair polariton scattering process with one of the polaritons dropping into the trap ground state is resonant.

${ }^{14}$ R. Huang et al., Phys. Rev. B 61, R7854 (2000).

${ }^{15}$ J. Erland et al., Phys. Rev. Lett. 86, 5791 (2001).

${ }^{16}$ R.M. Stevenson et al., Phys. Rev. Lett. 85, 3680 (2000).

${ }^{17}$ R. Houdré et al., Phys. Rev. Lett. 85, 2793 (2000).

${ }^{18}$ C. Ciuti et al., Phys. Rev. B 62, R4825 (2000).

${ }^{19}$ C. Benoit à la Guillaume et al., Phys. Rev. 177, 567 (1969); H. Haug et al., Phys. Status Solidi B 82, 531 (1977); S.W. Koch et al., ibid. 89, 43 (1978).

${ }^{20}$ P. Bigenwald et al., Phys. Rev. B 63, 035315 (2001), and references therein.

${ }^{21}$ I. Galbraith et al., J. Cryst. Growth 159, 667 (1996).

${ }^{22}$ R. Rapaport et al., Phys. Rev. Lett. 84, 1607 (2000).

${ }^{23}$ M. Skolnick, and R. Butté (Private communication).

${ }^{24}$ G. Malpuech et al., Appl. Phys. Lett. (to be published). 